\title{
An Asymptotic Expansion for Product Integration Applied to Cauchy Principal Value Integrals
}

\author{
P. Wesseling
}

Received March 17, 1975

Abstract. Product integration methods for Cauchy principal value integrals based on piecewise Lagrangian interpolation are studied. It is shown that for this class of quadrature methods the truncation error has an asymptotic expansion in integer powers of the step-size, and that a method with an asymptotic expansion in even powers of the step-size does not exist. The relative merits of a quadrature method which employs values of both the integrand and its first derivative and for which the truncation error has an asymptotic expansion in even powers of the step-size are discussed.

\section{Introduction}

Integrals of the following type are considered:

$$
I(f)=\int_{0}^{1} g(t) f(t) d t, \quad g(t)=\frac{h(t)}{t-\tau}, \quad \tau \in(0,1),
$$

where the integral is to be understood as a Cauchy principal value integral, and $f(t), h(t) \in C^{(p+1)}[0,1]$, with $p \geqq 1$. The function $h(t)$ is not included in $f(t)$, because in applications one often encounters integrals of the type (1.1), with $g(t)$ a fixed function, for example the kernel of an integral equation.

Hunter [3] has constructed a numerical quadrature formula for integrals of the type (1.1), which has an asymptotic expansion for the truncation error in even powers of the step-size. This makes a very effective application of Richardson extrapolation (Romberg integration) possible. Hunter's quadrature formula consists of the following generalised trapezoidal rule:

$$
T_{m}^{\prime}(f)= \begin{cases}\frac{1}{2 m}\left(g_{0} f_{0}+g_{m} f_{m}\right)+\frac{1}{m} \sum_{k=1}^{m-1} g_{k} f_{k}+\pi h(\tau) f(\tau) \cot m \pi \tau \quad \text { if } \tau \neq j / m, \\ \frac{1}{2 m}\left(g_{0} f_{0}+g_{m} f_{m}\right)+\frac{1}{m}\left\{\sum_{k=1}^{j-1}+\sum_{k=j+1}^{m-1}\right\} g_{k} f_{k} \\ +\frac{1}{m}\left(h^{\prime}(j / m) f(j / m)+h(j \mid m) f^{\prime}(j / m)\right) \quad \text { if } \tau=j / m,\end{cases}
$$

where $j$ is an integer $\in[1, m-1], f_{k}=f(k / m), g_{k}=g(k / m)$.

A disadvantage of Hunter's method is the need to evaluate $f^{\prime}(k / m)$. This is not always possible, for example when one solves an integral equation. Replacing the derivative by a divided difference introduces an additional truncation error with an asymptotic expansion in odd powers of $1 / m$, so that much of the effectiveness of Richardson extrapolation gets lost. Another disadvantage of Hunter's method is its sensitivity to rounding errors. 
Obviously it would be desirable to have a quadrature method for integrals of the type (1.1) which does not contain derivatives and which has an asymptotic expansion in even powers of $1 / \mathrm{m}$. We will investigate whether such a quadrature formula can be found within the class of product integration methods, or, if not, whether there are perhaps methods in this class which are less sensitive to rounding errors than Hunter's method.

\section{Derivation of Asymptotic Expansion for Product Integration}

De Hoog and Weiss [2] have derived asymptotic expansions for product integration with absolutely integrable singularities. Their analysis is adapted below to Cauchy integrals.

Let the Lagrangian interpolation polynomial $L_{k}(t)$ be defined by:

$$
\begin{aligned}
L_{k}(t) & =\frac{w(t)}{w^{\prime}\left(u_{k}\right)\left(t-u_{k}\right)}, \quad k=1(1) n ; \\
w(t) & =\prod_{k=1}^{n}\left(t-u_{k}\right) ; \quad 0 \leqq u_{1}<u_{2}<\cdots<u_{n} \leqq 1, \quad n \leqq p .
\end{aligned}
$$

The function $f(t)$ is approximated by the piecewise Lagrangian interpolation polynomial $f_{n}(t)$, defined as follows:

$$
\begin{aligned}
f_{n}(t) & =\sum_{k=1}^{n} L_{k}\left(m\left(t-t_{\ell}\right)\right) f\left(t_{\ell k}\right) ; \quad t_{\ell} \leqq t<t_{\ell+1}, \\
t_{\ell} & =\frac{\ell}{m}, \quad t_{\ell k}=t_{\ell}+u_{k} / m, \quad \ell=0(1) m-1, \quad k=1(1) n .
\end{aligned}
$$

The following product integration formula for (1.1) is considered:

$$
I_{m}(f)=\int_{0}^{1} g(t) f_{n}(t) d t
$$

The integral in (2.3) is a Cauchy principal value integral. For the existence of $I_{m}(f)$ it is necessary and sufficient that $f_{n}(t)$ be Hölder-continuous; in other words, we must have:

$$
n \geqq 2, \quad u_{1}=0, \quad u_{n}=1 .
$$

Eq. (2.3) can be written in the following form:

Writing :

$$
I_{m}(f)=\sum_{t=0}^{m-1} \sum_{j=1}^{n} q_{t j} f\left(t_{\ell j}\right)
$$

$$
\tau=\left(k+s_{0}\right) / m, \quad 0 \leqq s_{0}<1 \text {, with } k \text { an integer, }
$$

one obtains the following expression for the weights $q_{\ell j}$ :

$$
q_{\ell i}=\int_{0}^{1} h\left(\frac{\ell+s}{m}\right) L_{j}(s) \frac{1}{\ell+s-k-s_{0}} d s .
$$

If necessary, the foregoing integral is interpreted as a Cauchy principal value integral. An exception has to be made for the case $s_{0}=0, \ell=k-1, k$, because then the singularity lies at the boundary of the integration interval, a situation for which the Cauchy principal value integral is not defined. Except for $q_{k-1, n}$ 
and $q_{k, 1}$ the numerator contains a factor $\ell+s-k$, so that (2.7) still holds. One finds that $q_{k-1, n}$ and $q_{k, 1}$ must satisfy:

$$
q_{k-1, n}+q_{k, 1}=\int_{-1}^{1} h\left(\frac{k+s}{m}\right) L^{*}(s) \frac{1}{s} d s,
$$

with $L^{*}(s) \equiv L_{n}(s+1),-1 \leqq s<0 ; L^{*}(s) \equiv L_{1}(s), 0 \leqq s<1$. Note that because of $(2.4)$ the function $L^{*}(s)$ is Hölder-continuous, so that the preceding integral exists as a Cauchy principal value integral. The fact that (2.8) does not define $q_{k-1, n}$ and $q_{k, 1}$ separately is of no consequence, because both weights multiply the same function value $f\left(t_{k-1, n}\right)=f\left(t_{k, 1}\right)=f\left(t_{k}\right)$. One may arbitrarily define

$$
q_{k-1, n}=q_{k, \mathbf{1}} \text {. }
$$

This completes the definition of the weights in the quadrature formula (2.5).

The truncation error $E_{m}(f)$ is defined by:

$$
E_{m}(f)=I_{m}(f)-I(f)
$$

De Hoog and Weiss [2] prove, that

$$
E_{m}(f)=\sum_{r=0}^{p-n} m^{-n-r} \int_{0}^{1} w_{r}(s) \frac{1}{m} \sum_{l=0}^{m-1} g\left(t_{l}+s h\right) f^{(n+r)}\left(t_{l}+s h\right) d s+O\left(m^{-p-1}\right),
$$

where $w_{r}(s)=w(s) p_{r}(s)$, with $p_{r}(s)$ a polynomial of degree $r$, and $h=1 / m$.

In the derivation of (2.11) no use is made of the properties of $g(t)$, so that (2.11) holds not only for absolutely integrable $g(t)$, which is what [2] is concerned with, but also for $g(t)$ of the type $g(t)=h(t) /(t-\tau)$. Of course, if $\tau=k / m$ Eq. (2.11) does not make sense, because then the singularity lies at the boundary of two integration intervals. It is easily seen, that for $\tau=k / m$ (2.11) must be replaced by:

$$
\begin{aligned}
E_{m}(f)= & \sum_{r=0}^{p-n} m^{-n-r} \int_{0}^{1} w_{r}(s) \frac{1}{m}\left\{\sum_{l=0}^{k-2}+\sum_{t=k+1}^{m-1}\right\} g\left(t_{l}+s h\right) f^{(n+r)}\left(t_{l}+s h\right) d s \\
& +\sum_{r=0}^{p-n} m^{-n-r} \int_{-1}^{1} \Omega_{r}(s) g\left(t_{k}+s h\right) f^{(n+r)}\left(t_{k}+s h\right) d s+O\left(m^{-p-1}\right),
\end{aligned}
$$

where

$$
\Omega_{r}(s)=\left\{\begin{array}{l}
w_{r}(s), \quad s \geqq 0, \\
w_{r}(s+1), \quad s<0 .
\end{array}\right.
$$

The same result is obtained if (2.11) is interpreted in the following way, for $\tau=k / m$ :

$$
E_{m}(f)=\sum_{r=0}^{p-n} m^{-n-r} \lim _{\varepsilon \downarrow 0} \int_{\varepsilon}^{1-8} w_{r}(s) \frac{1}{m} \sum_{l=0}^{m-1} g\left(t_{l}+s h\right) f^{(n+r)}\left(t_{l}+s h\right) d s+O\left(m^{-p-1}\right) .
$$

In what follows we will base ourselves on (2.11), interpretated as a Cauchy principal value integral in the usual sense, or if $\tau=k / m$, as defined by (2.13).

We proceed to derive an asymptotic expansion for the generalized trapezoidal $\operatorname{sum} \sum_{l=0}^{m-1} g\left(t_{l}+s h\right) f^{(n+r)}\left(t_{l}+s h\right)$. The analysis proceeds along similar lines as 
the investigation of Lyness and Ninham [6] concerning generalized trapezoidal sums of absolutely integrable functions.

If $\phi(t)$ is a periodic generalised function with period 1 , then it can be represented by a Fourier series as follows (see for example Lighthill [5]):

$$
\phi(t)=\sum_{k=-\infty}^{\infty} c_{k} \exp (2 \pi i k t) .
$$

For the concept of a generalised function and the meaning of the (possibly in the ordinary sense divergent) Fourier series (2.14) the definitions of [5] are adopted. From (2.14) it follows, that

$$
\phi\left(t_{\ell}+s h\right)-c_{0}=\sum_{k=-\infty}^{\infty} c_{k} \exp \left(2 \pi i k\left(t_{\imath}+s h\right)\right)
$$

where the prime denotes omission of the term with $k=0$. It follows, that

$$
\frac{1}{m}\left(\sum_{i=0}^{m-1} \phi\left(t_{l}+s h\right)-c_{0}\right)=\sum_{k=-\infty}^{\infty} c_{k m} \exp (2 \pi i k(s-1)),
$$

where the subscript $k m$ means $k$ times $m$, and where use has been made of the fact, that

$$
\frac{1}{m} \sum_{i=0}^{m-1} \exp \left(2 \pi i k \frac{\ell+s}{m}\right)= \begin{cases}\exp (2 \pi i k(s-1) / m), & |k| / m=\text { integer } \\ 0 \quad, & \text { otherwise. }\end{cases}
$$

For $\phi(t)$ we choose successively the functions $\phi_{r}(t), r=O(1) p-n$, defined by:

$$
\phi_{r}(t)=g(t) f^{(n+r)}(t), \quad 0<t \leqq 1 ; \quad \phi_{r}(t+1)=\phi_{r}(t) .
$$

The Fourier coefficient $c_{k m}$ can be asymptotically approximated for large $m$ in the following way (see Lighthill [5]). In $(0,1] \phi_{r}(t)$ lacks derivatives at $t=\tau$ and $t=1$. The function $\phi_{r}(t)-F_{1, r}(t)$, with $F_{1, r}(t)=h(\tau) f^{(n+r)}(\tau) /(t-\tau)$, has an absolutely integrable $(p+1-n-r)$ th derivative in a neighbourhood of $t=\tau$. The same holds in a neighbourhood of $t=1$ for $\phi_{r}(t)-F_{2, r}(t)$, with

$$
F_{2, r}(t)=\frac{1}{2} \operatorname{sgn}(t-1) \sum_{j=0}^{p-n-r}\left(\phi_{r}^{(j)}(0)-\phi_{r}^{(j)}(1)\right) \frac{(t-1)^{j}}{j !} .
$$

The Fourier transforms of $F_{1, r}$ and $F_{2, r}$ are, respectively,

$$
\begin{aligned}
& G_{1, r}(y)=-h(\tau) f^{(n+r)}(\tau) \pi \operatorname{sign}(y) \exp (-2 \pi i \tau y), \\
& G_{2, r}(y)=\exp (-2 \pi i y) \sum_{i=0}^{p-n-r}\left(\phi_{r}^{(j)}(0)-\phi_{r}^{(j)}(1)\right)(2 \pi i y)^{-j-1} .
\end{aligned}
$$

The following asymptotic approximation of $c_{k m}$ is valid (Lighthill [5]):

One finds that

$$
c_{k m}=G_{1, r}(k m)+G_{2, r}(k m)+o\left(|k m|^{-p-1+n+r}\right) .
$$

$$
\begin{aligned}
& \sum_{k=-\infty}^{\infty} \exp (2 \pi i k(s-1)) G_{1, r}(k m)=2 \pi h(\tau) f^{(n+r)}(\tau) \sum_{k=1}^{\infty} \sin (2 \pi k(s-1-\tau m)) \\
& =\pi h(\tau) f^{(n+r)}(\tau) \cot (\pi(s-\tau m)) ;
\end{aligned}
$$


see Jones [4], p. 137. Furthermore,

$$
\begin{aligned}
& \sum_{k=-\infty}^{\infty} \exp (2 \pi i k(s-1)) G_{2, r}(k m) \\
& \quad=2 \sum_{j=0}^{p-n-r}\left(\phi_{r}^{(j)}(0)-\phi_{r}^{(j)}(1)\right)(2 \pi m)^{-i-1} \sum_{k=1}^{\infty} k^{-i-1} \cos \left(2 \pi k(s-1)-\frac{\pi}{2}(j+1)\right)(2, p) \\
& \quad=\sum_{j=0}^{p-n-r}\left(\phi_{r}^{(j)}(1)-\phi_{r}^{(j)}(0)\right) \frac{1}{(j+1) ! m^{i+1}} B_{j+1}(s-1)
\end{aligned}
$$

(see [1]), where $B_{j}(s)$ is the $j$-th Bernoulli polynomial as defined in [1].

Using (2.16), (2.18) and (2.22)-(2.24) one obtains:

$$
\begin{aligned}
& \frac{1}{m} \sum_{l=0}^{m-1} g\left(t_{l}+s h\right) f^{(n+r)}\left(t_{l}+s h\right)=\gamma_{r}+\pi h(\tau) f^{(n+\eta)}(\tau) \cot (\pi(s-\tau m)) \\
& \quad+\sum_{j=0}^{p-n-r}\left(\phi_{r}^{(j)}(1)-\phi_{r}^{(j)}(0)\right) \frac{1}{(j+1) ! m^{i+1}} B_{j+1}(s-1)+o\left(m^{-p-1+n+r}\right),
\end{aligned}
$$

where $\gamma_{r}=c_{0}$ in the Fourier-expansion of $\phi_{r}$. Substitution of (2.25) in (2.11) and collecting powers of $m$ one obtains:

$$
\begin{aligned}
E_{m}(f)= & m^{-n} \int_{0}^{1} w_{0}(s)\left(\gamma_{0}+\pi f^{(n)}(\tau) \cot \pi\left(s-s_{0}\right)\right) d s \\
& +m^{-p-1} \sum_{r=0}^{p-n} \int_{0}^{1} w_{r}(s)\left(\phi_{r}^{(p-n-r)}(1)-\phi_{r}^{(p-n-r)}(0)\right) \frac{B_{p-n-r+1}(s-1)}{(p-n-r+1) !} d s \\
& +m^{-n} \sum_{r=0}^{p-n-1} m^{-r-1} \int_{0}^{1}\left[w_{r+1}(s)\left(\gamma_{r+1}+\pi h(\tau) f^{(n+r+1)}(\tau) \cot \pi\left(s-s_{0}\right)\right)\right. \\
& \left.+\sum_{k=0}^{r} w_{r-k}(s)\left(\phi_{r-k}^{(k)}(1)-\phi_{r-k}^{(k)}(0)\right) \frac{B_{k+1}(s-1)}{(k+1) !}\right] d s+O\left(m^{-p-1}\right) .
\end{aligned}
$$

This is the desired asymptotic expansion for the truncation error. Because of the symmetry properties of the Bernoulli polynomials $B_{k}$ it is possible to choose $w(s)$ such that for $r$ even a number of the integrals above involving $B_{k}$ are zero so that if the cotangent were not present only even powers of $1 / m$ remain (cf. the Euler-MacLaurin expansion for the trapezoidal rule). However, it is obviously not possible to make the integral involving the cotangent vanish for any value of $r$ for arbitrary $s_{0}$.

\section{Discussion and Comparison with Hunter's Method}

It has just been shown that there is no product integration method (using piecewise Lagrangian interpolation) for Cauchy principal value integrals with an asymptotic expansion in even powers of $1 / \mathrm{m}$. Therefore one expects that if $f^{\prime}(\tau)$ can be evaluated Hunter's method coupled with Romberg integration will in general require much less computation time than a product integration method. If $f^{\prime}(\tau)$ is not known but is approximated by a difference, the asymptotic expansion for Hunter's method proceeds in powers of $1 / m$, as for product integration. Both methods profit equally from the application of Richardson extrapolation, and one expects that in general the computation times for the two methods will not differ greatly. 
With respect to rounding errors there is a significant difference between Hunter's method and product integration. For stability with respect to rounding errors it is necessary and sufficient that the sum of the absolute values of the weights is bounded. However, in Hunter's method (1.2) the weights $g_{k}$ are obviously not bounded, both as $\tau \rightarrow j / m$ for fixed $m$ and for $m \rightarrow \infty$ with fixed $\tau$, which makes this method sensitive to rounding errors in $f_{k}$. With product integration the weights are bounded, as is clear from (2.7), (2.8) and (2.9). Although for product integration the weights are bounded, the sum of their absolute values is not, because this quantity can be regarded as the product integration approximation to the divergent integral $\int_{0}^{1}|h(t) /(t-\tau)| d t$. We will determine the sum of the absolute values of the weights for the special case $n=2, u_{1}=0, u_{2}=1$, in order to find out how rapidly this sum diverges as $m \rightarrow \infty$. The case $n=2, u_{1}=0, u_{2}=1$ gives the simplest possible formula. This is probably the most important method for extrapolation purposes, because it seems that extrapolation methods applied to quadrature are most efficient when applied to simple, low-order formulae. With $n=2, u_{1}=0, u_{2}=1$ the following integration formula is obtained:

$$
I_{m}(f)=\sum_{l=0}^{m} \alpha_{l} f_{l}
$$

where $f_{\ell}=f(\ell / m), \alpha_{0}=-1+\beta_{1}-\beta_{0}-\ln (m \tau)$,

$$
\alpha_{m}=1+\beta_{m-1}-\beta_{m}+\ln |m(1-\tau)|, \quad \alpha_{\ell}=\beta_{\ell-1}-2 \beta_{\ell}+\beta_{\ell+1}, \quad \ell=1(1) m-1,
$$

$\beta_{\ell}=(\ell-m \tau) \ln |\ell-m \tau|$. For the sum of the absolute values of the weights one obtains:

$$
\sum_{\ell=0}^{m}\left|\alpha_{\ell}\right|=\left\{\begin{array}{l}
\ln ((1-\tau) / \tau), \quad 0<\tau<\sigma / m, \\
2+\ln \left(m^{2} \tau(1-\tau)\right)+2 \beta_{k}-2 \beta_{k+1}, \quad \sigma / m \leqq \tau \leqq 1-\sigma / m, \\
\ln (\tau /(1-\tau)), \quad 1-\sigma / m<\tau<1,
\end{array}\right.
$$

where $\sigma \approx 0.22$ is defined by the equation $(1-\sigma) \ln |(1-\sigma) / \sigma|=1$. For the derivation of (3.2), see the appendix.

For given $m$, the sum is bounded for $\tau$ in any closed subinterval of $(0,1)$, unlike the method of Hunter. For $m \rightarrow \infty$ the divergence is logarithmic. With Hunter's method the divergence is at least linear in $m$, because the sum contains weights that grow linearly: the weight $g_{k}$ contains a factor $(k / m-\tau)^{-1}$; the largest of these is at least $1 / m$.

As an example we compute the following integral, which has also been treated by Hunter:

$$
I=\int_{0}^{1} \frac{e^{-x} d x}{x-y}=e^{-y}\left\{E i(y)+E_{1}(1-y)\right\}, \quad 0<y<1,
$$

where $E i(y)$ and $E_{1}(y)$ are exponential integrals as defined by Abramowitz and Stegun [1]. The following table gives the numerical results for $y=0.375$ and $y=0.375+0.5 * 10^{-6}$, using (1.2) and (3.1). The computation of table 1 was performed with a mantissa of more than 16 decimal figures. 
Table 1.

\begin{tabular}{|c|c|c|c|c|}
\hline \multirow[b]{2}{*}{$m$} & \multicolumn{2}{|l|}{$y=0.375$} & \multicolumn{2}{|c|}{$y=0.375+0.5 * 10^{-6}$} \\
\hline & $T_{m}^{\prime}$ & $I_{m}$ & $T_{m}^{\prime}$ & $I_{m}$ \\
\hline 1 & -0.14466692 & -0.24238370 & -0.14466932 & -0.24238549 \\
\hline 2 & -0.25257520 & -0.33433314 & -0.25257697 & -0.33433472 \\
\hline 4 & -0.28938665 & -0.30481640 & -0.28938821 & -0.30481794 \\
\hline 8 & -0.30000672 & -0.30421905 & -0.26746681 & -0.30422003 \\
\hline 16 & -0.30279798 & -0.30385181 & -0.28652279 & -0.30385305 \\
\hline 31 & -0.30349036 & -0.30382794 & -0.30349183 & -0.30382942 \\
\hline 32 & -0.30350587 & -0.30376877 & -0.29536901 & -0.30377014 \\
\hline 63 & -0.30368161 & -0.30376350 & -0.30368308 & -0.30376497 \\
\hline 64 & -0.30368350 & -0.30374912 & -0.29961581 & -0.30375054 \\
\hline 127 & -0.30372773 & -0.30374789 & -0.30372920 & -0.30374936 \\
\hline 128 & -0.30372796 & -0.30374435 & -0.30169559 & -0.30374580 \\
\hline
\end{tabular}

Table 1 clearly exhibits the considerable influence of rounding errors in $T_{m}^{\prime}$ for $y=0.375+0.5 * 10^{-6}$. Note the large differences between the results for $m=31$ and $32, m=63$ and $64, m=127$ and 128 .

Hunter [3] has applied Romberg's method (repeated Richardson extrapolation) to $T_{m}^{\prime}$ for $y=0.375$, using the sequence $m=1,2,4,8,16,31,59$, and has obtained the value -0.30374278 .

Romberg's method was applied to the values of $T_{m}^{\prime}$ and $I_{m}$ listed in Table 1 using the sequence $m=2^{k-1}, k=1$ (1) 8 . The following results were obtained:

Table 2

\begin{tabular}{|c|c|c|c|c|}
\hline \multirow[b]{2}{*}{$k$} & \multicolumn{2}{|l|}{$y=0.375$} & \multicolumn{2}{|c|}{$y=0.375+0.5 * 10^{-8}$} \\
\hline & $T_{e, k}^{\prime}$ & $I_{e, k}$ & $T_{e, k}^{\prime}$ & $I_{\ell, k}$ \\
\hline 1 & -0.14466692 & -0.24238370 & -0.14466933 & -0.24238549 \\
\hline 2 & -0.28854463 & -0.36498295 & -0.28854619 & -0.36498446 \\
\hline 3 & -0.30253131 & -0.28497670 & -0.30253279 & -0.28497823 \\
\hline 4 & -0.30369083 & -0.30666738 & -0.25667658 & -0.30666801 \\
\hline 5 & -0.30374179 & -0.30348004 & -0.29580647 & -0.30348152 \\
\hline 6 & -0.30374277 & -0.30375612 & -0.29875313 & -0.30375753 \\
\hline 7 & -0.30374278 & -0.30374241 & -0.30126545 & -0.30374386 \\
\hline 8 & -0.30374278 & -0.30374279 & -0.30250595 & -0.30374425 \\
\hline
\end{tabular}

In this table $T_{e, k}^{\prime}$ and $I_{e, k}$ stand for the $k$-th entry on the main diagonal in Romberg's scheme.

As is to be expected, for $y=0.375$ the product-integration method converges somewhat more slowly than Hunter's method. For $y=0.375+0.5 * 10^{-6}$ rounding errors prevent convergence of Hunter's method. 


\section{Appendix: Derivation of Equation (3.2)}

For $\ell=1$ (1) $m-1$ the formula for $\alpha_{l}$ can be rewritten as

with $\gamma_{\ell}=\ell-k-s_{0}$, and

$$
\alpha_{1}=f\left(\gamma_{\ell}\right)
$$

$$
f(x)=x \ln \left|\left(x^{2}-1\right) / x^{2}\right|+\ln |(x+1) /(x-1)| .
$$

One finds that

$$
f^{\prime}(x) \geqq 0, \quad|x| \leqq 2^{-1 / 2}, \quad f^{\prime}(x) \leqq 0, \quad|x| \geqq 2^{-1 / 2} .
$$

Furthermore,

$$
f(-\infty)=f(0)=0 \text {. }
$$

From (A3 and (A4) it follows, that $f(x) \leqq 0, x \leqq 0$. Because $f(x)=-f(-x)$ it can be concluded that

Hence,

$$
\alpha_{\ell} \leqq 0, \quad \ell \leqq k ; \quad \alpha_{l}>0, \quad \ell>k, \quad \ell=1(1) m-1 .
$$

which results in:

$$
\sum_{\ell=1}^{m-1}\left|\alpha_{l}\right|=-\sum_{\ell=1}^{k} \alpha_{l}+\sum_{\ell=k+1}^{m-1} \alpha_{l}
$$

$$
\sum_{l=1}^{m-1}\left|\alpha_{l}\right|=\beta_{1}-\beta_{0}+2 \beta_{k}-2 \beta_{k+1}-\beta_{m-1}+\beta_{m}
$$

One may easily verify, that

$$
\begin{aligned}
& \alpha_{0} \geqq 0, \quad 0<\tau \leqq \sigma / m ; \quad \alpha_{0}<0, \quad \sigma / m<\tau<1 ; \\
& \alpha_{m} \geqq 0, \quad 0<\tau \leqq 1-\sigma / m ; \quad \alpha_{m}<0, \quad 1-\sigma / m<\tau<1 ;
\end{aligned}
$$

where $\sigma \approx 0.22$ is defined by the equation $(1-\sigma) \ln |(1-\sigma) / \sigma|=1$. Adding $\left|\alpha_{0}\right|$ and $\left|\alpha_{m}\right|$ to the sum in (A7) results in equation (3.2).

\section{References}

1. Abramowitz, M., Stegun, I. A. (Eds.): Handbook of Mathematical Functions. New York: Dover Publications Inc. 1965

2. De Hoog, F., Weiss, R.: Asymptotic expansions for product integration. Math. Comp. 27, 295-306 (1973)

3. Hunter, D. B.: The numerical evaluation of Cauchy principal values of integrals by Romberg integration. Numer. Math. 21, 185-192 (1973)

4. Jones, D. S.: Generalised Functions. London etc.: McGraw-Hill 1966

5. Lighthill, M. J.: Introduction to Fourier Analysis and Generalised Functions. Cambridge: Cambridge Univ. Press 1962

6. Lyness, J. N., Ninham, B. W.: Numerical quadrature and asymptotic expansions. Math. Comp. 21, 162-178 (1967)

P. Wesseling

Technische Hogeschool Twente

Onderafdeling der Toegepaste Wiskunde

Postbus 217

Enschede

The Netherlands 